\title{
Clinical value of fluorine-18 2-fluoro-2-deoxy-D-glucose positron emission tomography/computed tomography in penile cancer
}

\author{
Sheng Zhang ${ }^{1}$, Wenfeng $\mathbf{L i}^{2}$ and Fei Liang ${ }^{3}$ \\ ${ }^{1}$ Fudan University Shanghai Cancer Center, Department of Oncology, Shanghai Medical College, Fudan University, Shanghai, \\ China \\ 2 Department of Medical Oncology, Qingdao University Medical Center, Qingdao, China \\ ${ }^{3}$ Clinical Statistics Center, Shanghai Cancer Center, Fudan University, Shanghai, China \\ Correspondence to: Sheng Zhang, email: wozhangsheng@hotmail.com \\ Keywords: penile cancer; PET/CT; clinical impact \\ Received: February 08, $2016 \quad$ Accepted: May 09, $2016 \quad$ Published: May 14, 2016
}

\section{ABSTRACT}

\section{Purpose}

This study investigated the value of Fluorine-18 2-fluoro-2-deoxy-D-glucose (FDG) positron emission tomography (PET)/computed tomography (CT) imaging in the management of patients with advanced penile cancer.

Patients and Methods

Between January 2009 and August 2012, 48 patients with penile cancer at our center underwent FDG-PET/CT after CT $(n=39)$ or magnetic resonance imaging (MRI; $n=9$ ). The accuracy of FDG-PET/CT was assessed with both organ-based and patientbased analyses. FDG-PET/CT findings were validated by either biopsy or serial CT/ MRI. Clinician questionnaires performed before and after FDG-PET/CT evaluated whether the scan results affected management.

Results

One hundred fifteen individual lesions were evaluable in $\mathbf{4 2}$ patients for the organ-based analysis. Overall sensitivity was $85 \%$ and specificity was $86 \%$. In the patient-based analysis, overall sensitivity and specificity were $82 \%$ and $93 \%$, respectively. Pre- and post-PET surveys showed that FDG-PET/CT detected more malignant diseases than CT/MRI in 33\% patients. Planned treatments were changed in $57 \%$ patients after FDG-PET/CT scan.

Conclusion

FDG-PET/CT has good sensitivity and specificity in the detection of metastatic penile cancer. It provides more diagnostic information to enhance clinical management than CT/MRI.

\section{INTRODUCTION}

Squamous cell carcinoma of the penis is uncommon in developed countries, but its incidence in some developing countries is much higher $[1,2]$. The scarcity of this disease has limited the conduct of prospective studies evaluating the diagnosis, staging, treatment and follow-up.

Metastatic involvement of regional lymph nodes or distant organs is a strong prognostic factor for this disease and is associated with decreased survival [3]. The non-invasive imaging methods play important roles in this process. Fluorine-18 2-fluoro-2-deoxy-Dglucose (FDG) positron emission tomography (PET)/ $\mathrm{CT}$ provides anatomic and metabolic information for staging and restaging, and has been incorporated into the management of a variety of malignancies [4]. The use of FDG-PET/CT in patients with penile cancer may also help to characterize lesions that are uncertain by $\mathrm{CT}$ and/ or MRI. The role of PET imaging in penile cancer has not been adequately explored. One reason might be the urinary excretion of FDG interferes with visualization of the primary penile cancer and regional lymph nodes [5]. 
However, evaluation for metastatic lesions including local lymph nodes can be helpful in staging, treatment planning, and assessment of overall prognosis [6]. This study aims to explore the accuracy of PET/CT in detecting metastases using both patient-based and organ-based analysis and to identify the degree to which $\mathrm{PET} / \mathrm{CT}$ results affect clinical managements in patients with penile cancer.

\section{MATERIALS AND METHODS}

\section{Patient population}

Patients eligible for this study were prospectively registered in the PET Registry for Rare Cancer at Shanghai Cancer Center between January 2009 and August 2012. All patients had initial anatomic imaging with either CT or MRI followed by FDG-PET/CT.

\section{FDG-PET/CT}

${ }^{18}$ F-FDG was produced automatically by a cyclotron (RDS Eclips ST; Siemens) and an Explora FDG4 module (Siemens) in our center. All patients were required to fast for at least $6 \mathrm{~h}$ to ensure glucose blood levels below 10 $\mathrm{mmol} / \mathrm{L}$. Scanning was initiated $1 \mathrm{~h}$ after administration of the tracer $(7.4 \mathrm{MBq} / \mathrm{kg})$. The data acquisition procedure was as follows: CT was first performed, from the proximal thighs to head, with $120 \mathrm{kV}$, CARE Dose 4-dimensional mode, $80-250 \mathrm{~mA}$, and a pitch of 3.6. Immediately after CT, a PET emission scan that covered the identical transverse field of view was obtained. The acquisition time was 2-3 min per table position. PET image datasets were reconstructed iteratively by application of the CT data for attenuation correction, and coregistered images were displayed on a workstation.

\section{Data analysis}

The first goal was to investigate the sensitivity and specificity of FDG-PET/CT in identifying metastatic lesions in patients with penile cancer. Lesions recorded in FDG-PET/CT reports were catalogued and assessed further using histopathology from biopsies or serial subsequent imaging studies as the standard of reference. Biopsies were obtained at the discretion of the referring oncologist. Two types of correlations were performed: an organ-based analysis and a patient-based analysis. All FDG-PET/CT findings were classified as true positive, true negative, or false negative in both the organ-specific lesion analysis and the patient-based analysis. Two experienced physicians from nuclear medicine department independently evaluated the data to determine the status of lesions. If there was any discrepancy, a third physician will help determine the status of lesions.

A lesion was considered to be true positive if it was detected on PET/CT and subsequently confirmed to be cancerous by either biopsy or serial imaging with $\mathrm{CT}$ or MRI. A lesion seen on initial CT or MRI was considered a true negative if it was not detected on PET/CT and validated as benign by biopsy or serial imaging. A finding was considered false positive if suspicious FDG uptake was described on PET/CT but the biopsy was negative or subsequent serial imaging studies did not show evidence for malignancy, such as increase in size. A lesion was considered a false negative if it was not detected by PET/ CT but was initially seen on CT or MRI and subsequent imaging studies showed increase in size or if biopsy findings confirmed malignancy.

\section{Patient-based analysis}

The patient-based analysis was performed in a manner as other studies. In brief, all lesions were classified as true positive, true negative, false positive, or false negative. In the event of a discordant finding, a truepositive lesion superseded all other lesions including false negative, true negative, and false positive. Therefore, if a patient had at least one true-positive lesion, the PET/ CT scan was considered true positive. In the absence of a true-positive lesion, a false-negative lesion superseded a true-negative or a false-positive lesion. So if the PET/ $\mathrm{CT}$ was false negative in at least one disease site, it was considered to be a false negative overall.

\section{Clinical impact analysis}

The questionnaires on intended patient management, completed by urologic oncologists in our center, were collected before and after FDG-PET/CT to determine how the findings affected patient management as the previous studies [7]. The pre-FDG-PET/CT survey collected information regarding the indication for the scan and the clinician's management plan if it was not available. The post-FDG-PET/CT survey collected information on the clinician's planned management with the available scan results and whether the $\mathrm{PET} / \mathrm{CT}$ intervention avoided further testing. The managements included observation, additional imaging, tissue biopsy or needle inspiration, surgical treatment with curative intent, chemotherapy treatment with curative intent, chemotherapy with palliative intent, radiation therapy, and supportive care. The data were collected prospectively with the consent of the patients. The physician's answers on the surveys were confirmed by medical chart review.

\section{RESULTS}

Forty-eight patients with penile cancer were 
Table 1: Patient demographics and clinical characteristics

\begin{tabular}{|l|l|l|l|}
\hline Demographic or Clinical Characteristic & \multicolumn{2}{|l|}{$\begin{array}{l}\text { No. of Patients }(\boldsymbol{N}= \\
\text { 48) }\end{array}$} & \% \\
\hline Age, years & & & \\
\hline Median & & 56.6 & \\
\hline Range & & $29-77$ & \\
\hline ECOG performance status & & & \\
\hline 0 & 19 & & 39.6 \\
\hline 1 & 23 & & 48 \\
\hline 2 & 6 & & 12.5 \\
\hline Primary tumor intact & 11 & & 23 \\
\hline Skin ulceration & 19 & & 40 \\
\hline Lymph node stations clinically involved & & & \\
\hline Groin only (stage III) & 11 & & 23 \\
\hline Deep inguinal or pelvic (stage IV) & 28 & & 58.3 \\
\hline Distant metastasis & 21 & & 43.7 \\
\hline History of smoking & 40 & & 83 \\
\hline Current & 6 & & 12.5 \\
\hline Former & 34 & & 70.8 \\
\hline Prior treatment & & & \\
\hline Chemotherapy & 19 & & 39.6 \\
\hline Radiation therapy & 5 & & 10.4 \\
\hline Presentation of disease & & & \\
\hline Primary & 17 & & 35.4 \\
\hline Recurrent & 31 & & 64.6 \\
\hline
\end{tabular}

Abbreviations: ECOG, Eastern Cooperative Oncology Group.

included in this study (Table 1). And forty-two of these patients were evaluable for analysis. Six patients did not have further imaging studies after the FDG-PET/ $\mathrm{CT}$ and were excluded. The patient population involved distant metastases (44\%) and the remaining patients had groin/deep inguinal or pelvic lymph node metastases. The reasons for FDG-PET/CT are initial staging in 35\%, restaging or suspected recurrence in $65 \%$ of patients. Forty percent patients had received prior chemotherapy, and $10 \%$ received radiation therapy. Eighty-six percent of the findings considered suspicious for cancer on FDGPET/CT had an SUV $\geq 2.5$. For the other $15 \%$ lesions with SUV less than 2.5, the radiologist categorized those as suspicious malignancy.

\section{Organ-based analysis}

Organ-based analysis was performed on 115 lesions in the 42 evaluable patients (Table 2). The predominant site of disease was lymph node (47\%), followed by lung $(22 \%)$ and other organs. For the organ-based analysis, the overall FDG-PET/CT sensitivity was $85 \%$, and specificity was $86 \%$.

\section{Patient-based analysis}

After the PET/CT scans, the patients were followed by either a biopsy $(n=19)$ or follow-up scan $(n=23)$. The sensitivity was $75 \%$ in those with a follow-up scan and $83 \%$ in biopsy group (Table 3 ). The total sensitivity is $81 \%$ and the total specificity is $93 \%$.

\section{Clinical impact analysis}

Forty-four questionnaires were available for analysis. The survey reported that more disease was found on FDG-PET/CT compared with conventional CT or MRI in $33 \%$ of the patients. And less disease was found with PET/CT in $17 \%$ patients. $62 \%$ of the patients were stated to avoid more tests because of PET/CT (Figure 1).

The survey also showed that treatment change occurred in $57 \%$ of the patients (Table 4). It was reported that additional imaging was avoided after PET/CT scan, and the need for biopsy was negated in $16 \%$ of the patients. In patients planned for locoregional treatment, $18 \%$ were found to have distant metastases after PET/CT and thus changed to systemic chemotherapy. Altogether, $57 \%$ patients had their treatment changed based on the 
Table 2: Organ-specific lesion-based analysis of suspicious FDG-PET/CT uptake

\begin{tabular}{|l|l|l|l|l|l|l|}
\hline & \multicolumn{2}{l}{ Sites } & \multicolumn{2}{l|}{ Sensitivity } & \multicolumn{2}{l|}{ Specificity } \\
\hline Site of Disease & No. & $\mathbf{\%}$ & $\mathbf{\%}$ & $\mathbf{9 5 \%} \mathbf{C I}(\mathbf{\%})$ & $\%$ & $\mathbf{9 5 \%} \mathbf{C I}(\mathbf{\%})$ \\
\hline Lymph node & 54 & 47 & 93 & 72 to 99 & 85 & 60 to 94 \\
\hline Lung & 25 & 22 & 86 & 62 to 95 & 84 & 62 to 98 \\
\hline Bone & 18 & 15 & 90 & 65 to 100 & 100 & 66 to 100 \\
\hline Liver & 7 & 6 & 56 & 28 to 90 & 100 & 51 to 100 \\
\hline Soft tissue & 6 & 5 & 100 & 45 to 100 & 80 & 44 to 100 \\
\hline Adrenal & 3 & 3 & 100 & 34 to 100 & 100 & 45 to 100 \\
\hline Kidney & 2 & 2 & 100 & 21 to 100 & 100 & 28 to 100 \\
\hline Total sites & 115 & & 85 & 70 to 95 & 86 & 76 to 94 \\
\hline
\end{tabular}

Abbreviations: FDG, fluorine-18 2-fluoro-2-deoxy-D-glucose; PET, positron emission tomography; CT, computed tomography.

Table 3: Patient-based analysis of suspicious lesions reported by FDG-PET/CT

\begin{tabular}{|c|c|c|c|c|c|c|c|}
\hline \multirow[b]{3}{*}{ Validation } & \multicolumn{5}{|c|}{\begin{tabular}{|l|} 
No. of Lesions \\
\end{tabular}} & & \\
\hline & \multirow[b]{2}{*}{ Total } & \multirow{2}{*}{ True Positive } & \multirow{2}{*}{ False Positive } & \multirow{2}{*}{ False Negative } & \multirow{2}{*}{$\begin{array}{l}\text { True } \\
\text { Negative }\end{array}$} & Sensitivity(\%) & \multirow[t]{2}{*}{$\begin{array}{l}\text { Specificity } \\
(\%)\end{array}$} \\
\hline & & & & & & & \\
\hline Follow-up scans & 23 & 9 & 1 & 3 & 11 & 75 & 91 \\
\hline Biopsy & 19 & 15 & 0 & 3 & 1 & 83 & 100 \\
\hline Total & 42 & 23 & 1 & 5 & 14 & 82 & 93 \\
\hline
\end{tabular}

Abbreviations: FDG, fluorine-18 2-fluoro-2-deoxy-D-glucose; PET, positron emission tomography; CT, computed tomography.

Table 4: Patient management changes based on FDG-PET/CT results

\begin{tabular}{|l|l|l|}
\hline & \multirow{2}{*}{ No. of Patients $(\boldsymbol{N}=\mathbf{4 4})$} & \\
\cline { 2 - 4 } & & $\mathbf{\%}$ \\
\hline Physician Changes & 7 & 16 \\
\hline Additional imaging avoided & 7 & 16 \\
\hline \multirow{2}{*}{ Locoregional treatment changed to metastatic treatment } & 8 & 18 \\
\hline Surveillance changed to treatment & 2 & \\
\hline Local radiotherapy changed to chemotherapy & 1 & 5 \\
\hline Total management changes & 25 & 2 \\
\hline
\end{tabular}

Abbreviations: FDG, fluorine-18 2-fluoro-2-deoxy-D-glucose; PET, positron emission tomography; CT, computed tomography.

results of PET/CT (Table 4). The change of treatment caused by $\mathrm{PET} / \mathrm{CT}$ was confirmed by medical chart review.

Including patients for whom the plan before PET/ CT was another type of imaging (eg, CT or MRI) may have overestimated the impact of PET/CT on the patient management change. As previously, an image-adjusted impact was performed and 7 patients were excluded. The management change based on $\mathrm{PET} / \mathrm{CT}$ results was $41 \%$ of the patients.

\section{DISCUSSION}

In our study, FDG-PET/CT showed excellent sensitivity and specificity in the detection of metastases in patients with advanced penile cancer. The majority of patients in this study were undergoing restaging or evaluation for suspected recurrence, a clinical scenario that was suitable for the use of FDG-PET/CT since the likelihood of metastases for this group of patients would be high. The sensitivity and specificity of FDG-PET/CT in this disease were similar to those in other epithelial malignancies [8, 9]. This study did not include the patients with superficial disease alone. Prior studies do 


\section{The extent of disease on FDG PET/CT compared with CT or MRI}

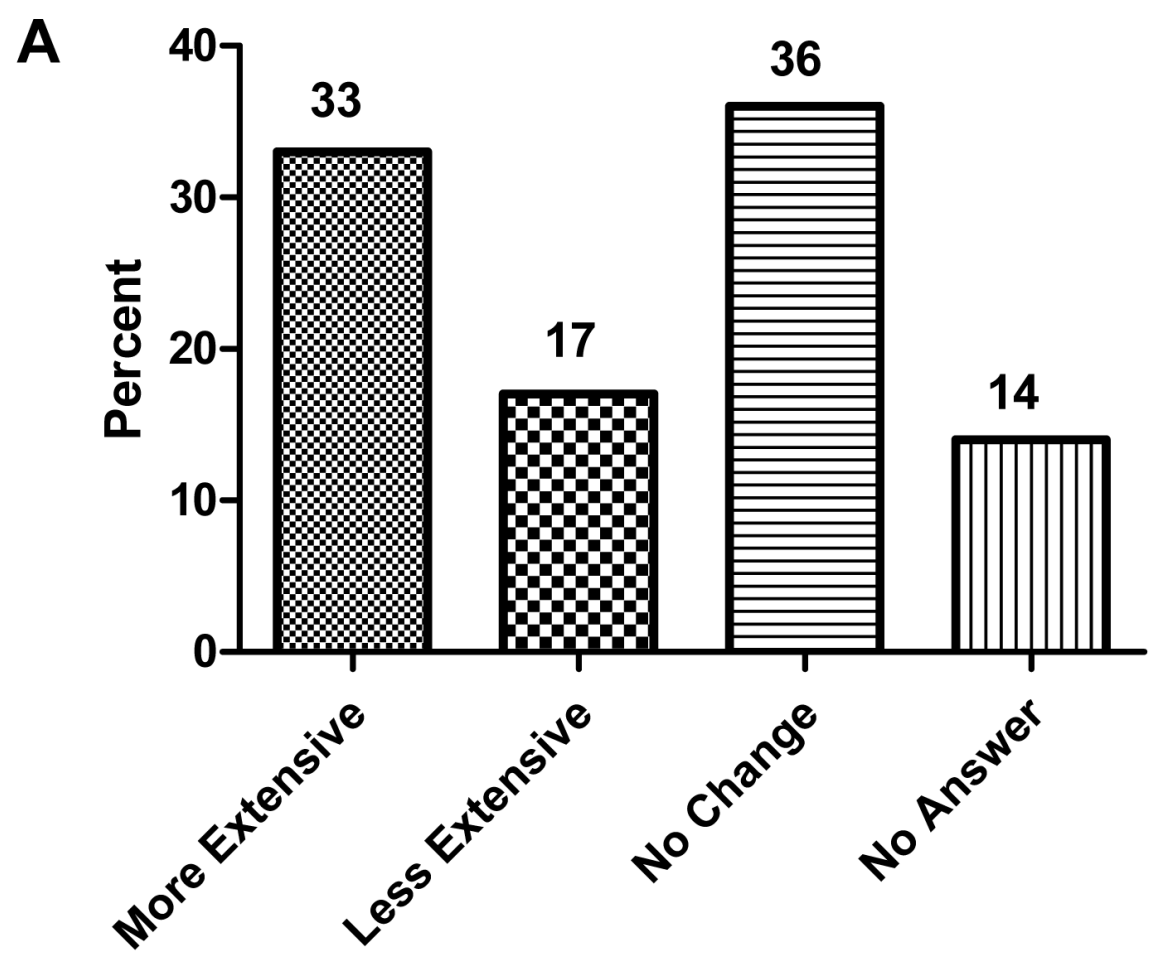

Did FDG PET/CT avoid more tests?

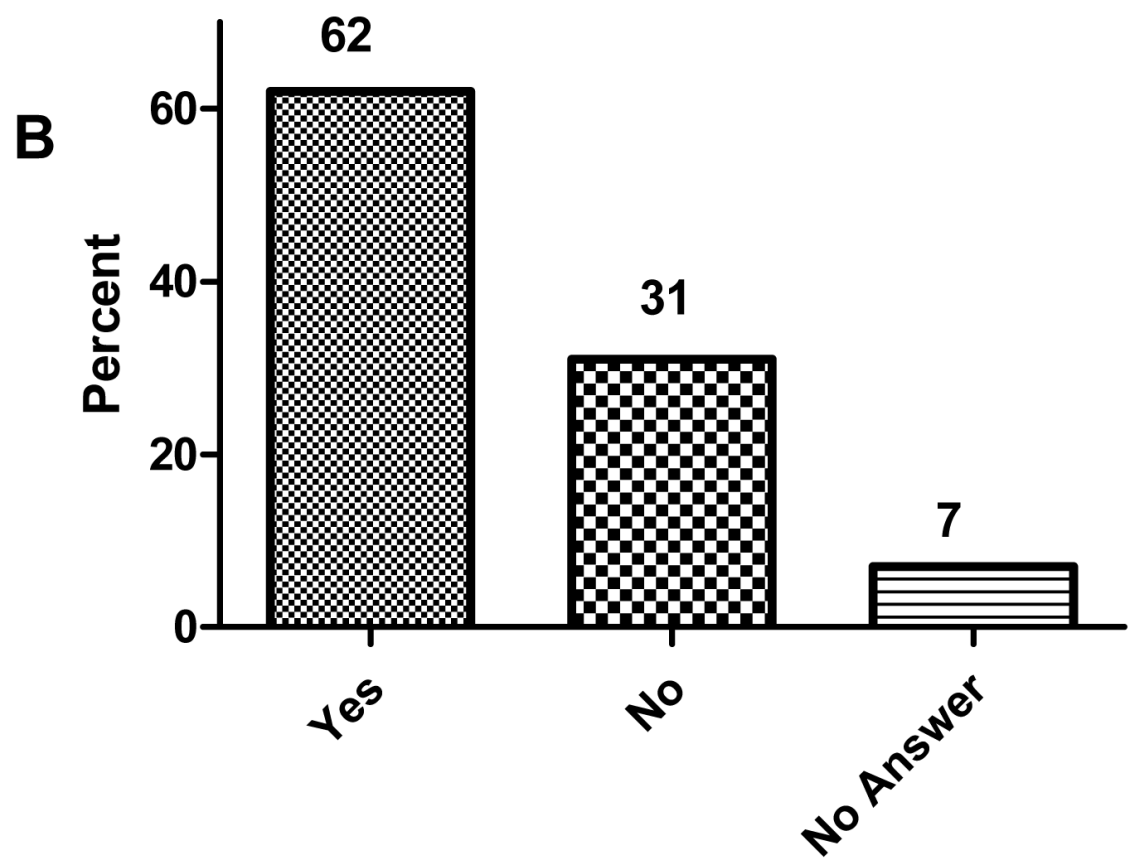

Figure 1: Physician answers $(n=44)$ to questionnaires concerning extent of disease and the impact of fluorine-18 2-fluoro-2-deoxy-D-glucose (FDG)PET/computed tomography (CT) on clinical management. Responses were confirmed by medical record review. A. Responses to extent of disease on FDG-PET/CT compared with CT or magnetic resonance imaging (MRI). B. Responses to FDG-PET/CT and avoidance of additional tests. 
not support routine $\mathrm{PET} / \mathrm{CT}$ in this setting since metastatic involvement of regional lymph nodes or distant sites is rare in superficial diseases [10-12].

The presence and extent of regional lymph and distant metastases are among the most decisive prognostic factors in penile cancer. For a group of locally advanced penile cancer, a recent phase II trial has demonstrated that the neoadjuvant chemotherapy could elicit a clinically meaningful response of $50 \%$. Overall survival was also significantly associated with chemotherapy responsiveness. Thus, a standard neoadjuvant medical intervention for this group of patients has been established $^{13}$. Our results here showed that the results of $\mathrm{PET} / \mathrm{CT}$ could be useful for staging and evaluation of the patients before the decision of treatment management.

Previously, there were several studies which explored the role of $\mathrm{PET} / \mathrm{CT}$ in penile cancer patients $[10-12,14,15]$. These studies were performed mainly in small sample size or retrospectively with wide variability in sensitivity and specificity. A recent pooled-analysis has shown that PET/CT has low sensitivity in $\mathrm{cN} 0$ patients for detection of regional lymph node involvement in penile cancer patients. However, patients with clinically palpable lymph node may benefit from PET/CT since the sensitivity in this subgroup of patients is high [16]. The results of our study are concordant here.

Another feature in this study is the questionnaire used to determine the assessment of clinical utility for PET/CT. 57\% patients were deemed by treating physicians to have derived benefit from FDG-PET/CT. 7 biopsies were avoided. Although pathologic confirmation remains the gold standard, biopsy is not always possible because of the risk with lesions deep in the pelvis near vascular structures, or patient refusal. In these instances, FDGPET/CT may serve as a useful substitute to assess the suspected site for the treatment choice. The use of PET/ CT could possibly avoid further testing, unnecessary invasive procedures, or inadequate therapy, as has been demonstrated in other malignancies.

There are some limitations in our study. Selection bias may have occurred since only patients with likelihood for recurrent/metastatic disease were referred for a PET/CT scan. Only when there is clinical suspicion of abnormality on standard cross-sectional imaging such as CT or MRI, PET/CT can be ordered. The limited number of patients in this study might be a concern. However, to our knowledge, this is the largest study so far assessing the role of PET/CT in penile cancer for this rare disease.

In summary, this study demonstrated that FDG$\mathrm{PET} / \mathrm{CT}$ could be useful in the detection of metastases for patients with advanced penile cancer. It may provide better clinical information for the plan or change of treatment management.

\section{ACKNOWLEDGMENTS}

We gratefully thank the staff members in the Department of Medical Oncology and urology center at Fudan University Cancer Center for their suggestions and help. We also thank Dr Chao Ma from nuclear department for the assistance.

\section{CONFLICTS OF INTEREST}

None.

\section{GRANT SUPPORT}

None.

\section{REFERENCES}

1. Jemal A, Siegel R, Ward E, Hao Y, Xu J and Thun MJ. Cancer statistics, 2009. CA Cancer J Clin. 2009; 59:225249.

2. Misra S, Chaturvedi A and Misra NC. Penile carcinoma: a challenge for the developing world. Lancet Oncol. 2004; 5:240-247.

3. Pizzocaro G, Algaba F, Horenblas S, Solsona E, Tana S, Van Der Poel H and Watkin NA. EAU penile cancer guidelines 2009. Eur Urol. 2010; 57:1002-1012.

4. Xu G, Zhao L and He Z. Performance of whole-body PET/ $\mathrm{CT}$ for the detection of distant malignancies in various cancers: a systematic review and meta-analysis. J Nucl Med. 2012; 53:1847-1854.

5. Scher B, Seitz M, Reiser M, Hungerhuber E, Hahn K, Tiling R, Herzog P, Schneede P and Dresel S. 18F-FDG PET/CT for staging of penile cancer. J Nucl Med. 2005; 46:14601465.

6. Kibel AS, Dehdashti F, Katz MD, Klim AP, Grubb RL, Humphrey PA, Siegel C, Cao D, Gao F and Siegel BA. Prospective study of $[18 \mathrm{~F}]$ fluorodeoxyglucose positron emission tomography/computed tomography for staging of muscle-invasive bladder carcinoma. J Clin Oncol. 2009; 27:4314-4320.

7. Hillner BE, Siegel BA, Liu D, Shields AF, Gareen IF, Hanna L, Stine SH and Coleman RE. Impact of positron emission tomography/computed tomography and positron emission tomography (PET) alone on expected management of patients with cancer: initial results from the National Oncologic PET Registry. J Clin Oncol. 2008; 26:21552161.

8. Hoekstra CJ, Stroobants SG, Smit EF, Vansteenkiste J, van Tinteren H, Postmus PE, Golding RP, Biesma B, Schramel FJ, van Zandwijk N, Lammertsma AA and Hoekstra OS. Prognostic relevance of response evaluation using [18F]-2fluoro-2-deoxy-D-glucose positron emission tomography in 
patients with locally advanced non-small-cell lung cancer. J Clin Oncol. 2005; 23:8362-8370.

9. Apolo AB, Riches J, Schoder H, Akin O, Trout A, Milowsky MI and Bajorin DF. Clinical value of fluorine-18 2-fluoro-2-deoxy-D-glucose positron emission tomography/ computed tomography in bladder cancer. J Clin Oncol. 2010; 28:3973-3978.

10. Rosevear HM, Williams H, Collins M, Lightfoot AJ, Coleman T and Brown JA. Utility of (1)(8)F-FDG PET/CT in identifying penile squamous cell carcinoma metastatic lymph nodes. Urol Oncol. 2012; 30:723-726.

11. Souillac I, Rigaud J, Ansquer C, Marconnet L and Bouchot O. Prospective evaluation of (18)F-fluorodeoxyglucose positron emission tomography-computerized tomography to assess inguinal lymph node status in invasive squamous cell carcinoma of the penis. J Urol. 2012; 187:493-497.

12. Schlenker B, Scher B, Tiling R, Siegert S, Hungerhuber E, Gratzke C, Tilki D, Reich O, Schneede P, Bartenstein $\mathrm{P}$, Stief CG and Seitz M. Detection of inguinal lymph node involvement in penile squamous cell carcinoma by 18F-fluorodeoxyglucose PET/CT: a prospective singlecenter study. Urol Oncol. 2012; 30:55-59.
13. Pagliaro LC, Williams DL, Daliani D, Williams MB, Osai W, Kincaid M, Wen S, Thall PF and Pettaway CA. Neoadjuvant paclitaxel, ifosfamide, and cisplatin chemotherapy for metastatic penile cancer: a phase II study. J Clin Oncol. 2010; 28:3851-3857.

14. Graafland NM, Valdes Olmos RA, Teertstra HJ, Kerst JM, Bergman AM and Horenblas S. 18F-FDG PET/CT for monitoring induction chemotherapy in patients with primary inoperable penile carcinoma: first clinical results. Eur J Nucl Med Mol Imaging. 2010; 37:1474-1480.

15. Graafland NM, Leijte JA, Valdes Olmos RA, Hoefnagel CA, Teertstra HJ and Horenblas S. Scanning with 18F-FDG$\mathrm{PET} / \mathrm{CT}$ for detection of pelvic nodal involvement in inguinal node-positive penile carcinoma. Eur Urol. 2009; 56:339-345.

16. Sadeghi R, Gholami H, Zakavi SR, Kakhki VR and Horenblas S. Accuracy of 18F-FDG PET/CT for diagnosing inguinal lymph node involvement in penile squamous cell carcinoma: systematic review and meta-analysis of the literature. Clin Nucl Med. 2012; 37:436-441. 